Service social

\title{
Un doctorat francophone en service social
}

\section{Yves Hurtubise}

Volume 36, numéro 1, 1987

L’informatique dans les services sociaux

URI : https://id.erudit.org/iderudit/706336ar

DOI : https://doi.org/10.7202/706336ar

Aller au sommaire du numéro

Éditeur(s)

École de service social de l'Université Laval

ISSN

1708-1734 (numérique)

Découvrir la revue

Citer ce document

Hurtubise, Y. (1987). Un doctorat francophone en service social. Service social, 36(1), 5-8. https://doi.org/10.7202/706336ar d'utilisation que vous pouvez consulter en ligne.

https://apropos.erudit.org/fr/usagers/politique-dutilisation/ 


\section{ÉDITORIAL}

\section{Un doctorat francophone en service social}

Dix ans après en avoir parlé une première fois, cinq ans après avoir formé un comité sur le sujet et après avoir obtenu l'autorisation de six niveaux hiérarchiques, l'École de service social de l'Université Laval est enfin en mesure de recevoir le premier groupe d'étudiants au doctorat en service social. ${ }^{1}$

Le processus est long et souvent méconnu, même de ceux qui ont gardé des contacts privilégiés avec l'université. Entre l'évidence d'un besoin, sa démonstration, la préparation d'un dossier circonstancié et l'autorisation d'agir, il y a plusieurs univers. Celui d'abord des scientifiques, de ceux qui se croient capables, non seulement de prouver le besoin mais aussi de proposer un programme, de montrer noir sur blanc que les ressources sont disponibles et compétentes. Il y a aussi l'univers des gestionnaires et des « politiques » qui demandent, voire exigent, preuves sur preuves, engagements sur engagements. À ce niveau de discussion, l'enveloppe a autant sinon plus d'importance que son contenu. Il faut savoir parler science, gestion, politique et ne pas être trop mauvais stratège.

Quand un dossier comme celui-ci se rend à terme, c'est-à-dire quand il y a des personnes dûment inscrites au programme et assises dans une salle de cours, il convient de saluer bien bas celles et ceux qui ont eu le courage, la patience, l'énergie, la détermination de mener à bien l'entreprise.

Imagine-t-on qu'il s'agit ici d'une première dans le monde francophone? Certes, le mérite est moins grand quand on sait que la France universitaire commence à peine à faire une petite place au travail social. Par contre, en prenant le Québec comme unité de mesure, on s'aperçoit que les huit unités de formation universitaire en travail social n'offraient pas de programme de doctorat; au Canada, un seul est offert, à l'Université de Toronto. Plus de quarante ans après la création de la première école de service social au Québec, un programme complet de troisième cycle est créé. 
La portée d'un tel programme est non négligeable. Dans le système universitaire, les études de doctorat sont celles qui exigent de l'étudiant la production de nouvelles connaissances. Cet enjeu est majeur pour la personne qui s'y engage. Il est déterminant pour celles et ceux qui œuvrent dans le même secteur d'activités.

Pour une école de service social, l'introduction de ce niveau d'études est un objet de fierté, disons-le sans fausse modestie. Cela comporte aussi des défis importants à relever. J'en proposerai quatre ici. Le premier est de réussir à créer un milieu propice à l'exploration intellectuelle. Il s'agit de rassembler les conditions favorables à l'échange d'idées. Or, nulle unité de formation universitaire ne peut prétendre à elle seule refléter toutes les tendances théoriques que l'on retrouve en service social.

Il y a ici une obligation d'ouverture sur ces tendances, donc sur les autres universités, tant québécoises que canadiennes et étrangères. On viendra étudier au doctorat à Laval parce qu'il y aura là un milieu intellectuel en effervescence, un brassage d'idées, une culture. C'est ma conviction que nous ne relèverons ce défi qu'à la condition d'accueillir chez nous celles et ceux qui réfléchissent sur la société. Pourtant, la tradition de collaboration interuniversitaire est faible. À ce niveau, il y a de nouvelles pratiques à développer, de nouvelles zones d'échanges à créer. Certes, nous pouvons compter sur nos expériences antérieures, dans le cadre de l'Association canadienne des écoles de service social par exemple, ou dans le cadre plus récent du Regroupement des unités de formation universitaire en travail social du Québec, sans compter les échanges plus ponctuels amorcés par l'un ou l'autre des professeurs. Tout ceci est bien mince, me semble-t-il, par rapport à l'enjeu de créer un nouvel espace intellectuel concernant le service social. II faudra développer de nouveaux types d'échanges, vaincre les timidités, dépasser les antagonismes, accepter les différences idéologiques. L'ouverture d'un programme de doctorat invite à créer ce nouvel espace, mais elle nous fait aussi l'obligation d'une présence accrue dans les débats sociaux : c'est là le deuxième défi.

Le service social s'est développé, chez nous comme ailleurs, à partir d'une pratique auprès des populations aux prises avec des problèmes sociaux. Nous avons eu du mal à assumer notre fonction de critique sociale, avec le résultat que nous sommes encore relativement absents des grands enjeux sociaux. Cette absence avait été constatée au moment des travaux de la Commission Castonguay-Nepveu. Notre présence est maintenant plus importante au moment de la Commission Rochon. Mais qu'avons-nous eu à dire au moment du Livre blanc sur la fiscalité des particuliers (qui annonçait une approche différente des allocations sociales) ? Qu'avons-nous eu à proposer comme analyse au moment de 
la publication du Livre vert sur le logement? Y a-t-il une contribution particulière que nous pouvons apporter au moment où l'État-Providence est remis en question, au moment où une nouvelle philosophie politique se met en place? Bien sûr, on trouvera des textes, des analyses, des réflexions provenant de personnes intimement associées au service social.

Mais plus globalement, quelle est notre contribution, à titre d'intellectuels, dans ces questions qui touchent à l'avenir de notre société? Pour le dire en clair, nous sommes à la frange des débats, notre apport est marginal, loin de ce que nous sommes en mesure de faire. $\mathrm{Si}$ produire des docteurs dans un secteur d'activités signifie produire des connaissances nouvelles, celles-ci devront avoir une résonnance sociale, apporter des éclairages nouveaux, confronter les acteurs sociaux, faire avancer la société vers une meilleure connaissance d'elle-même et des contradictions qui la travaillent. Produire des docteurs en service social, c'est être présent au cœur des questions, organiser des débats, des colloques, des congrès; publier des analyses, non seulement pour un public d'initiés, mais aussi pour celles et ceux qui vivent les problèmes.

Un autre défi touche à la pratique du travail social. Les praticiens du service social sont en droit d'attendre des productions qui contribueront au renouvellement de leur pratique et à un nouvel éclairage sur celleci. Produire une thèse de doctorat n'est pas qu'un exercice de cueillette de données, c'est surtout un effort d'en proposer une interprétation. C'est exploiter avec rigueur des matériaux dont on fera un amalgame pour donner un sens à la réalité.

Au moment où la pratique professionnelle est de plus en plus encadrée - déqualifiée, déconsidérée, disent certains, - il apparaît important, voire urgent, de multiplier les recherches, d'inviter à l'analyse, de faire appel à l'imagination pour préciser les conditions optimales d'exercice du travail social.

Offrir un programme de doctorat, c'est aussi être capable d'accueillir des personnes qui viennent d'univers culturels variés. Déjà, de nombreux professeurs et chercheurs étrangers viennent pour des périodes plus ou moins longues constater sur place ce que nous faisons. Plusieurs professeurs et chercheurs d'ici vont ailleurs chercher des idées nouvelles, un ressourcement nécessaire. Ces échanges s'amplifieront sans doute grâce à l'existence d'un programme de doctorat ; il me semble qu'on doive aller plus loin et susciter ces échanges, lancer des invitations, de telle sorte que nous travaillions de moins en moins dans un univers fermé mais, au contraire, pleinement conscients des idées et des problèmes de ceux et celles qui partagent notre condition d'humains.

Ces quatre défis posent, en particulier à l'École de service social de I'Université Laval, des questions énormes. Comment faire tout cela 
alors que les effectifs professoraux sont déjà surchargés? Comment développer la nécessaire collaboration interuniversitaire alors que la tradition et la philosophie politique dominante incitent à la compétition ? Comment faire en sorte que ce nouveau programme ne devienne pas l'affaire de quelques éminences grises?

Les professeurs de notre École ont abordé ces questions, ont commencé à réfléchir à de nouvelles façons d'exercer leur travail. Il ne peut y avoir de recettes miracles, on l'aura deviné. L'École de service social de l'Université Laval devra rendre des comptes aux gestionnaires et aux "politiques». Elle le devra aussi aux autres pour qui la création d'un doctorat en service social est également importante.

École de service social,

\section{Yves HURTUBISE}

Université Laval.

\section{Note}

1 Un dépliant d'information est disponible à notre École. Le lecteur intéressé peut en faire la demande au directeur du programme. 